\title{
Lösungsmöglichkeiten nach fehlgeschlagenen Osteosynthesen am Oberschenkelschaft
}

\author{
Ulrich Wiebking, Christian Krettek
}

\section{Zusammenfassung}

Für die Versorgung von Frakturen im Bereich des Oberschenkelschaftes hat sich in den letzten Jahrzehnten mehr und mehr die Methode der Oberschenkelmarknagelung etabliert. Es wird in diesem Beitrag daher auf Lösungsmöglichkeiten nach Fehlschlägen dieser Osteosyntheseform näher eingegangen. Auch für diese etablierte Methode der Oberschenkelmarknage- lung ergeben sich Fehlermöglichkeiten, die das ursprüngliche Ziel einer frühzeitig belastungsstabilen Osteosynthese in den Hintergrund drängen können. Zugang, Reposition, Implantat und Beingeometrie bilden die wesentlichen Komponenten für Erfolg oder Misserfolg. Anhand prägnanter Beispiele aus dem klinischen Alltag wird versucht, hier eine Hilfestellung zur Lösung von Problemen in diesen Bereichen zu geben.

\section{Einleitung}

Die Oberschenkelmarknagelung von Schaftfrakturen hat sich als ein Standardverfahren etabliert. Wesentlichen Anteil daran hat die Tatsache, dass diese Stabilisierungsmethode hohe Heilungsraten und geringe Komplikationsraten aufweist [2]. Aber auch diese Methode bietet Möglichkeiten für Fehlschläge. In diesem Zusammenhang steht auch die Tatsache einer nicht unerheblichen Lernkurve bei Anwendung dieses zunächst in seiner Anwendung einfach erscheinenden Implantates. Die Hauptprobleme ergeben sich dabei vor allem im Bereich der Beingeometrie (Rotation und Abweichungen in der Frontalebene), der Zugangswahl, der Implantatentfernung und des Verlustes an Reposition. Die Frage der Lösung dieser Probleme in diesem Bereich stellt häufig eine zusätzliche Schwierigkeit dar und bewegt sich meist abseits des „Lehrbuchwissens“. Im Folgenden sollen für die angesprochenen Problembereiche bei der Versorgung von Oberschenkelschaftfrakturen exemplarisch Lösungsmöglichkeiten dargestellt werden.

OP-JOURNAL 2004; 20: 150-157

(c) Georg Thieme Verlag KG Stuttgart · New York

\section{Hauptteil}

Lösungsmöglichkeiten bei Fehlschlägen in der Beingeometrie

Während die Achsabweichung in der sagittalen Ebene bei den Oberschenkelmarknagelungen von geringerer Bedeutung ist, stellt die Abweichung in der Frontalebene und die Rotationsfehler den Operateur häufiger vor Probleme. Bereits im Operationsaal können aber die nötigen Maßnahmen durchgeführt werden, diesen Problemen effektiv zu begegnen. $[5,6]$. Zur Kontrolle der Achse in der Frontalebene dient beispielsweise die Kabelmethode, wobei die zu analysierenden Strukturen streng im Zentrum des Strahlenfeldes abgebildet werden müssen (Abb.1).

Zur Kontrolle der intraoperativen, radiologischen Bestimmung der Femurrotation kann weiterhin das Trochanter-minor-Zeichen, das Kortikalissprungzeichen und das Durchmesserdifferenzzeichen herangezogen werden (Abb.2 u.3).

Die intraoperative Kontrolle der Rotation und Achse ist schnell und einfach durchführbar (Trochanter-minor-, Kortikalissprung- und Durchmesserdifferenzzeichen, Kabelmethode), und erspart ggf. Enttäuschungen bei der postoperativen Röntgenbildanalyse.

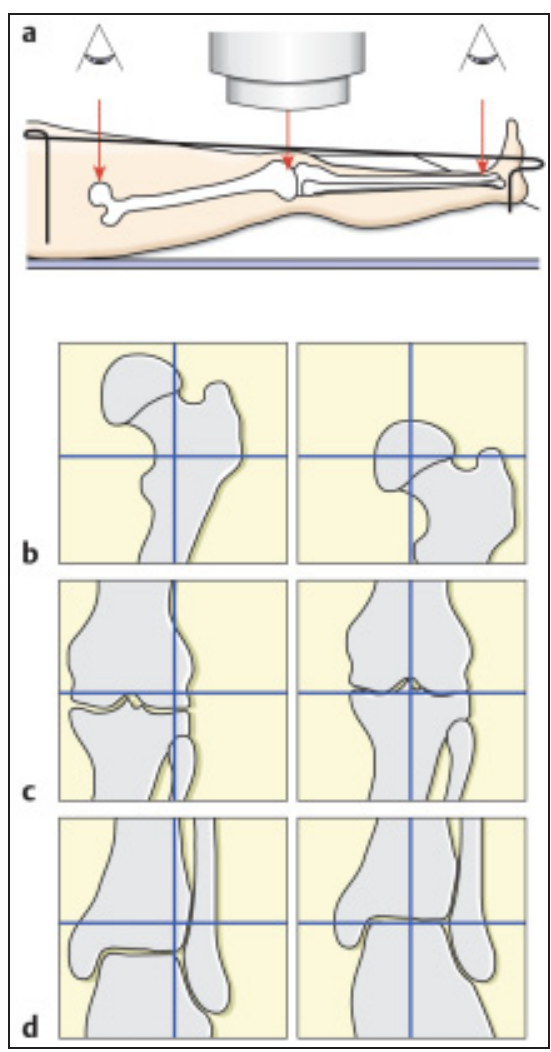

Abb.1a-d Kabeltechnik zur Achsenkontrolle in der Frontalebene. Das Knie ist gestreckt und die Patella zeigt nach ventral. In dieser Position wird der Winkel zwischen medialem Fußrand und der Vertikalen abgeschätzt und notiert. Schritt 1: Mit dem Bildverstärker in streng vertikaler Ausrichtung und dem Zentrum des Femurkopfes im Zentrum des Bildverstärkers wird das Femurkopfzentrum mit dem Stift auf der Haut markiert. Schritt 2: In analoger Technik wird das Zentrum des Taluskorpus auf Haut oder Abdeckmaterial markiert. Die Assistenz spannt dann das Kabel des Elektrokauters zwischen beiden Landmarken. Schritt 3: Das Kniegelenk wird im Bildverstärker dargestellt. Die Abweichung des projizierten Elektrokauterkabels vom Zentrum des Kniegelenks gibt Hinweise auf die Achsabweichung in der Frontalebene. 


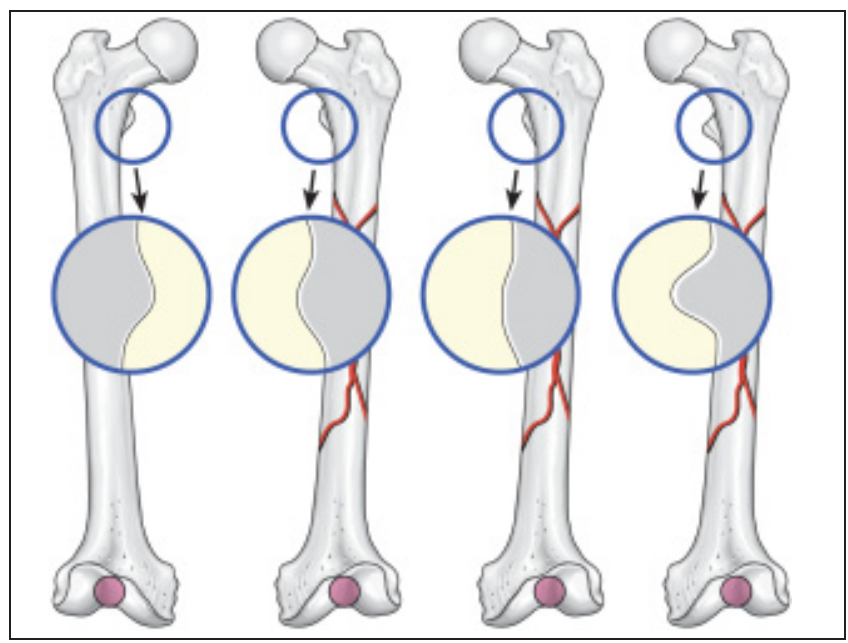

Abb.2 Technik zur intraoperativen Rotationskontrolle. Präoperativ wird der Trochanter minor der kontralateralen Seite mit dem Bildverstärker im Single-shot-Modus bei streng nach ventral zeigender Patella $\left(90^{\circ}\right.$-Beugung im Knie) abgebildet und im Bildspeicher gehalten. Bei korrekter Rotationseinstellung entspricht das ipsilaterale Abbildungsprofil dem kontralateralen Muster. Drehfehler nach außen (AR) oder innen (IR) haben charakteristische Trochanter-minor-Abbildungsprofildifferenzen.

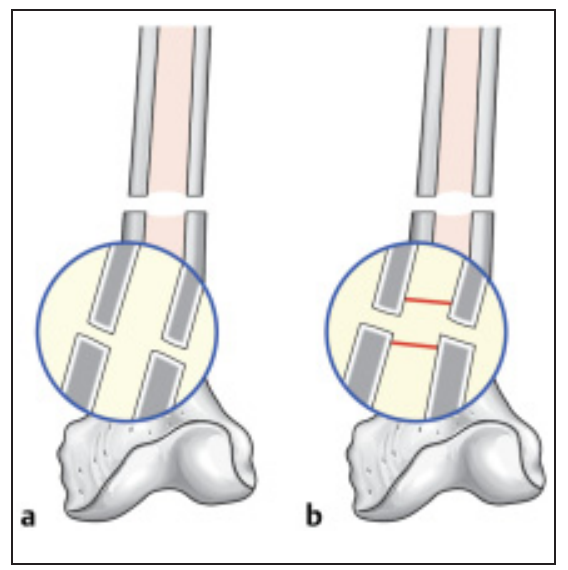

Abb.3au.b Kortikalissprung- und Durchmesserdifferenzzeichen als Hinweis auf das Vorhandensein eines Rotationsfehlers. Positiv nur bei Vorhandensein großer Rotationsfehler. (a) Kortikalissprungzeichen: Bei vorhandenen Drehfehlern können die kortikalen Strukturen des proximalen und distalen Hauptfragmentes mit unterschiedlicher Dicke abgebildet werden. (b) Durchmesserdifferenzzeichen: Dieses Zeichen ist positiv in den Fällen, wo das Knochenrohr ovale und nicht runde Konfiguration aufweist. In diesen Fällen werden proximales und distales Hauptfragment mit unterschiedlichen Querdurchmessern abgebildet.

Trotz Anwendung dieser Kontrollen intraoperativ, können sich z.B. durch die für die spezielle Situation ungeeignete Implantate auch sekundäre Rotationsfehlstellungen einstellen [4]. Der Effekt der geringen Torsionssteifigkeit verrie- gelter geschlitzter Marknägel geringen Durchmessers wird im nachfolgend beschriebenen Fall besonders deutlich. Ein damals 20-jähriger Patient hatte im Rahmen eines Pkw-Unfalles eine Oberschenkelschaftfraktur links erlitten. Die Versorgung erfolgte zunächst auswärts mittels verriegeltem AO-Universalmarknagel $12 \mathrm{~mm}$ (Abb.4a,b).

Postoperativ zeigte sich im Verlauf eine Verkürzung des Beins um $3 \mathrm{~cm}$, ein Außenrotationsfehler von $36^{\circ}$ und eine Valgusfehlstellung sowie anhaltende Beschwerden, die eine Rückkehr zu sportlichen Aktivitäten verhinderten. Bei näherer Analyse der Röntgenbilder war der Eintrittspunkt des Nagels zu weit lateral gewählt worden. In der daraufhin durchgeführten Korrekturosteotomie wurde die Achse in der Frontal- und Axialebene korrigiert und durch einen gebohrten, geschlitzten und verriegelten AO-Universalnagel komplettiert (Abb.4cu.d). Der Patient stellte sich 3 Jahre nach diesem Eingriff erneut mit Beschwerden in der linken Hüfte, Knie und Sprunggelenk vor. Es bestand ein Beckenschiefstand, eine Beinverkürzung von $13 \mathrm{~mm}$ links und ein Außendrehfehler von $41^{\circ}$ im CT (Abb.4e-g). Die durchgeführte derotierende Schrägosteotomie ermöglichte die Korrektur des Drehfehlers und eine Verlängerung der Beinlänge links um 10 mm und wurde durch eine Plattenosteosynthese (LC-DCP) gehalten (Abb.4h u.i i). Der Außendrehfehler betrug postoperativ $6,8^{\circ} \mathrm{im} \mathrm{CT}$. Drei Jahre später erfolgte die Metallentfernung bei perstistierenden Schmerzen über dem medialem Kondylus aber sonst klinischer Beschwerdefreiheit. Die Anwendung eines geschlitzten dünnen Nagels für die erste Korrekturosteomie setzte eine genügend lange Verklemmungsstrecke voraus, die hier aber nicht gegeben war. Dadurch konnten die angreifenden Torsionsmomente auf einer langen Strecke des geschlitzten $\mathrm{Na}-$ gels wirken und bewirkten somit einen erneuten Drehfehler.

Kann auf einen Aufbohrvorgang bei der Marknagelung verzichtet werden, sollten torsionssteife Implantate, wie ungeschlitzte Marknägel oder Platten zur Stabilisierung einer Korrekturosteotomie verwendet werden.

\section{Lösungsmöglichkeiten bei Fehlern im Zugangsbereich}

Häufig verursacht ein Fehler Folgeprobleme, die den ursprünglich korrekten Plan zunichte machen können. Eindrücklich zeigt sich die Folge des nicht korrekt gewählten Nageleintrittspunktes auf die Stabilität des Knochen-Implantat-Komplexes und somit auf die Geometrie bei einer nach initialer auswärtiger durch einen unverriegelten AO-Universalmarknagel versorgten dislozierten Oberschenkelfraktur (32 B2) (Abb.5a-d). Das Implantat hätte aufgrund der langen Knochenrohrstrecke proximal und seines Durchmessers auch ohne Verriegelung ausreichend Verklemmung gehabt. Der nicht korrekt gewählte Nageleintrittspunkt führte aber zum Ausbruch des proximalen Fragmentes. Somit war eine ausreichende Verklemmung nicht mehr gewährleistet und bei dann fehlender Verriegelung zeigte sich wenige Tage später ein ausgeprägtes Teleskoping. Die Richtigkeit dieser Überlegungen wurde durch den Verfahrenswechsel auf einen zur Ausheilung führenden verriegelten AOUniversalmarknagel bestätigt.

\section{Lösungsmöglichkeiten bei Implantatproblemen}

Der einer zyklischen Belastung ausgesetzte Marknagel versagt irgendwann, wenn die Beanspruchungen die Ausdauergrenzen überschreiten. Die Faktoren, welche dieses Rennen zwischen Frakturheilung und Ermüdung beeinflussen, sind die Stabilität des Knochenimplantatverbundes (Teilabstützung vorhanden oder fehlend), eine verzögerte Knochenbruchheilung oder Pseudarthrose und das Rehabilitationsprogramm und Kör- 

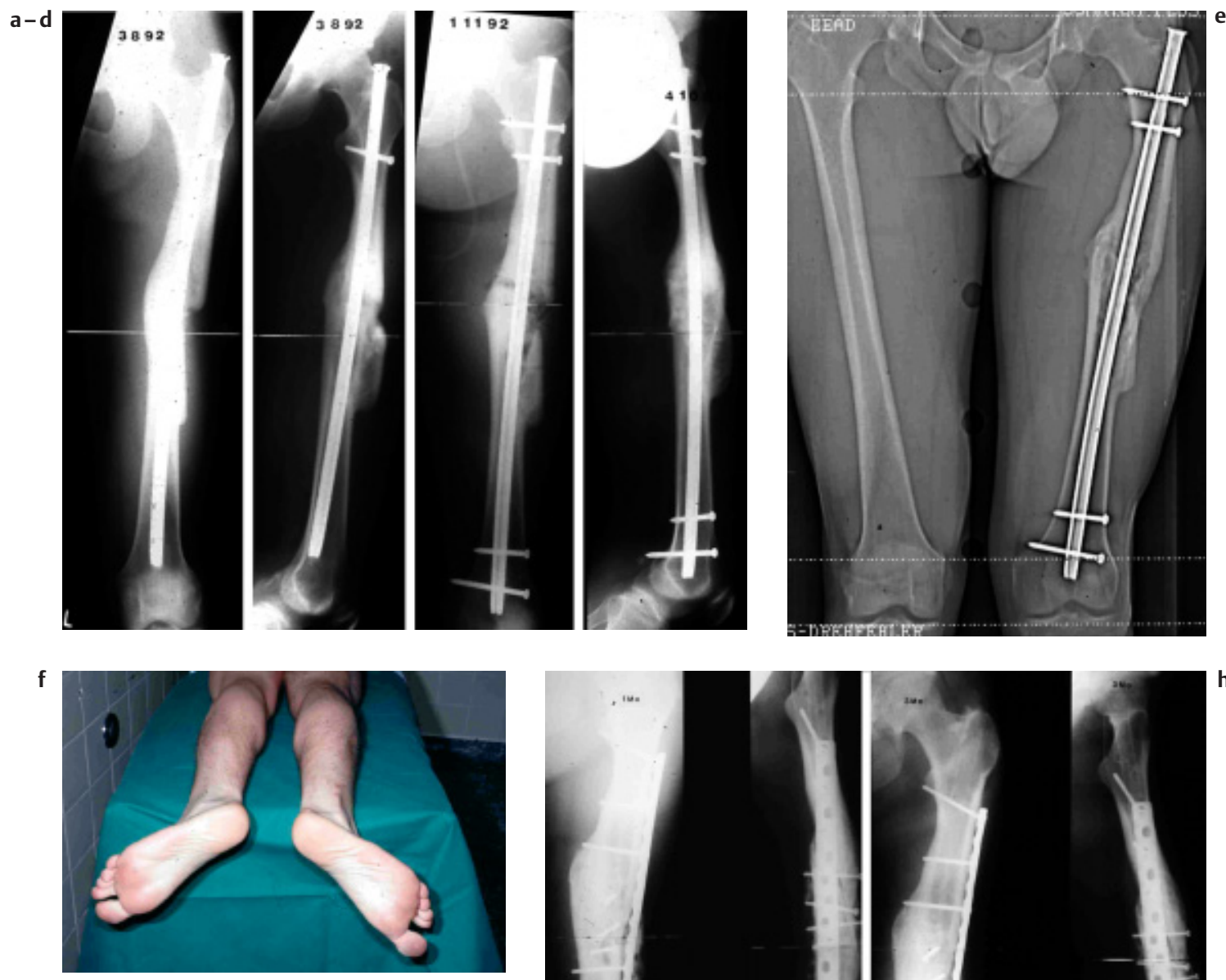

g
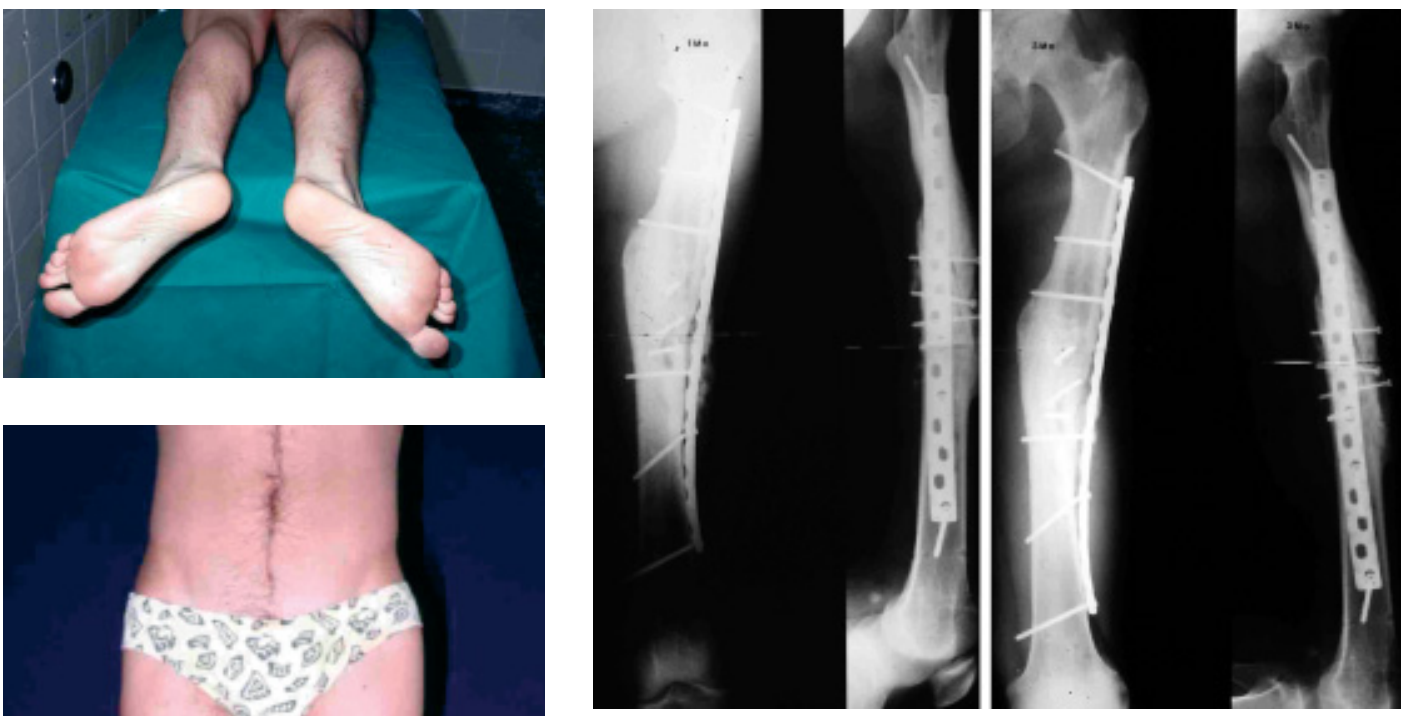

$h, i$

Abb.4a-i 20 Jahre, männlich, Oberschenkelschaftfraktur 32 B2. (a) Primärversorgung durch AO-Universalmarknagel, a.-p. (b) lateral. (c) Korrekturosteotomie 1 Jahr später mit AO-Universalnagel, ungeschlitzt, a.-p. (d) lateral. (e) Drehfehler CT 3 Jahre später mit Außenrotationsfehler $39^{\circ}$. (f) Klinische Darstellung des Außendrehfehlers links. (g) Beinverkürzung links von $13 \mathrm{~mm}$ nach Brettchenmethode. (h) Re-Korrekturosteotomie mit LC-DCP, a.-p. (i) lateral.

pergewicht $[1,3,10]$. Die Methoden, um das distale Fragment eines zerbrochenen, hohlen Nagels zu entfernen, hängen von der Passierbarkeit von Haken oder Schrauben entlang des Zentrums des Nagels ab [8]. Bei geschlitzten und hohlen Implantaten bieten sich immer mehrere Optionen zur Metallentfernung. Für solide Implantate sind die Möglichkeiten beschränkt. Eine Lösung für dieses Problem ist anhand eines distal gebrochenen Femurmarknagels dargestellt.
Der 24-jährige Patient erlitt im Rahmen eines Hochrasanztraumas mit seinem Pkw eine geschlossene Femurschaftfraktur links (AO 32 B3) (Abb.6a). Die Fraktur verlief dabei direkt unterhalb des Isthmus. Die Versorgung erfolgte mittels unaufgebohrter Marknagelung (420/9, Synthes, Ti-6Al-7Nb) und proximaler wie auch distaler zweifacher Verriegelung $(4,9 \mathrm{~mm}$ ) (Abb. 6b). Aufgrund weiterer Verletzungen u.a. der rechten Seite (Femurkondylenfraktur rechts, Tibiakopf- 
a

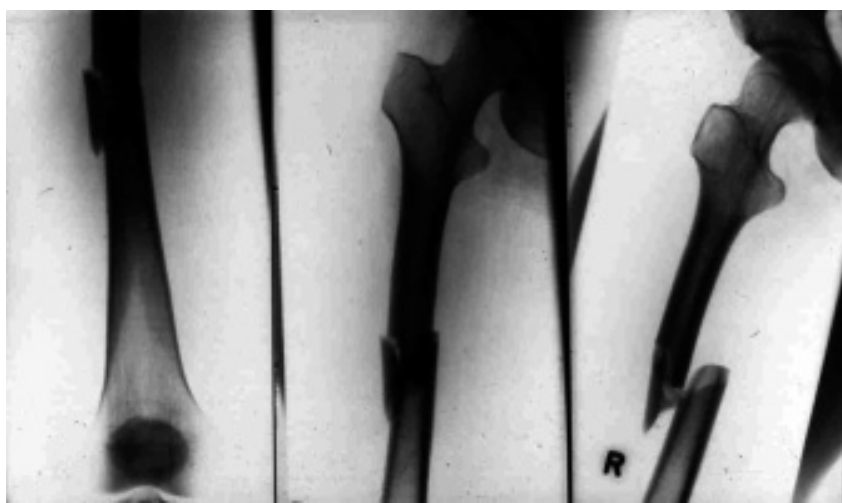

$b-d$
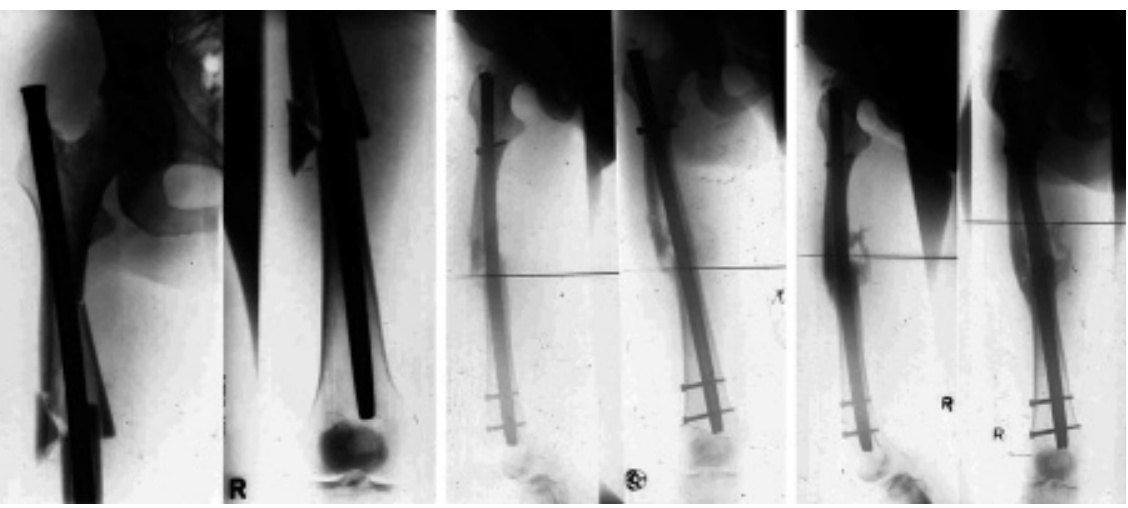

Abb.5a-d: Teleskoping nach Bruch des proximalen Fragmentanteils als Folge eines falschen Eintrittpunktes. (a) Unfallbild 32 B2. (b) Teleskoping nach wenigen Tagen. (c) Röntgenkontrolle nach Versorgung mit verriegeltem AO-Universalmarknagel. (d) Konsolidierung nach 6 Monaten.
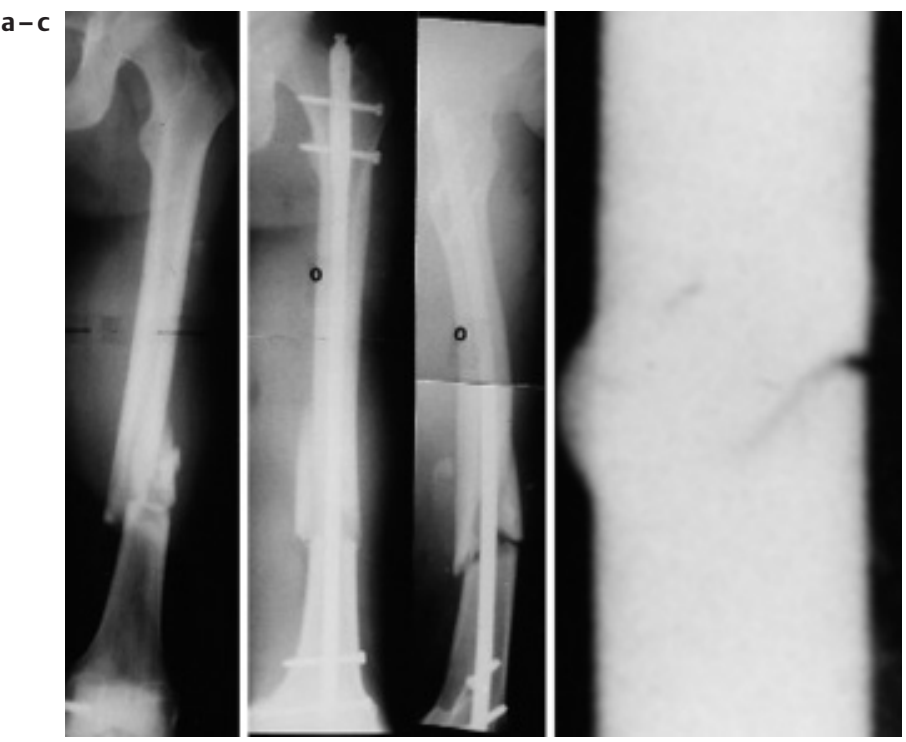

Abb.6a-c (a) Präoperative Röntgenaufnahme, die die infraisthmale Fraktur des femoralen Schafts zeigt. Die Schraube im medialen-femoralen Kondylus war während einer vorherigen Instandsetzung des hinteren Kreuzbandes implantiert worden. (b) Röntgenaufnahme nach Einfügung eines soliden Titanlegierungsnagels mit statischer Verriegelung mit Schrauben. Die Fraktur wurde in geringfügiger Distraktion retiniert. (c) Vergrößerung der seitlichen Röntgenaufnahme vom Ende des Nagels neun Wochen postoperativ, die einen vollständigen Bruch durch den Nagel an den Proximaleren von den zwei distalen Schraubenlöchern zeigen. Das Schraubenloch kann nicht gesehen werden, weil es von der Schraube überdeckt wird. fraktur rechts, Ellenbogenluxation rechts) wurde erst nach 42 Tagen die Bettruhe aufgehoben. Während dieser Zeit wurde intensive Physiotherapie beider unterer Extremitäten durchgeführt. Sechs Wochen nach dem Eingriff wurde der Patient an Unterarmgehstützen unter Teilbelastung der rechten Extremität weiter mobilisiert. Röntgenaufnahmen vom linken Oberschenkelknochen nach sechs Wochen zeigten keine Verschiebung des Bruchs und beginnende Kallusbildung. In der neunten Woche begann der Patient ohne vorhergehendes Trauma über Instabilitätsgefühle des linken Beins zu klagen. Röntgenaufnahmen zeigten, dass der $\mathrm{Na}$ gel im Bereich oberhalb der distalen Schraubenlöcher durchgebrochen war $(\mathbf{A b b} .6 \mathbf{c})$. Unter Anwendung einer modizifierten Rückschlagtechnik gelang die Bergung des distalen Nagelrestes und die erneute Marknagelung.

Der proximale Nagelanteil wurde in herkömmlicher Weise entfernt (Abb.7a). Um den distalen Abschnitt zu entfernen, wurde ein $4 \mathrm{~cm}$ großer, longitudinaler Zugang über dem seitlichen femoralen Kondylus bis auf den Knochen geschaffen. Unter BV-Kontrolle wurde ein Führungsdraht in den seitlichen femoralen Kortex platziert, etwa zwei Zentimeter proximal der lateralen Gelenkfläche und vor dem lateralen Kollateralband. Er verlief dabei im spongiösen Knochen schräg abwärts auf die beiden Schraubenlöcher zu. Mit dem kanülierten Eröffnungsinstrument aus dem UTN-Instrumentarium wurde dann ein kortikospongiöser Zylinder herausgeschnitten. Somit war ein seitlicher „Arbeitskanal“" geschaffen (Abb.7bu.c), über den nun nach Vergrößerung mittels einer scharfen Kürette, das Nagelreststück mittels Dorn und Hohmann-Haken nach dem „Schuhanzieherprinzip“ im Markraum hochgehebelt werden konnte (Abb.7d-f). Von proximal wurde ein $9 \mathrm{~mm}$-Markraumnagel eingeführt, der die Führung von oben ermöglichte (Abb. 7g).

Auf diese Weise gelang die Entfernung des Nagelrestes. Der kortikospongiöse Zylinder wurde zum Verschluss des $\mathrm{Ar}$ beitskanals wieder eingesetzt und anschließend die Operation durch Implantation eines 420/9-Femurnagel (Synthes) ohne Aufbohrung abgeschlossen. Der Innenrotationsfehler von $20^{\circ}$ wurde dadurch ebenfalls korrigiert. Die Operationszeit betrug $110 \mathrm{~min}$, die Durchleuchtungszeit 2,39 min. Postoperativ wurde der Patient vor Übergang zur Vollbelastung zunächst mit $20 \mathrm{~kg}$ Teilbelastung 

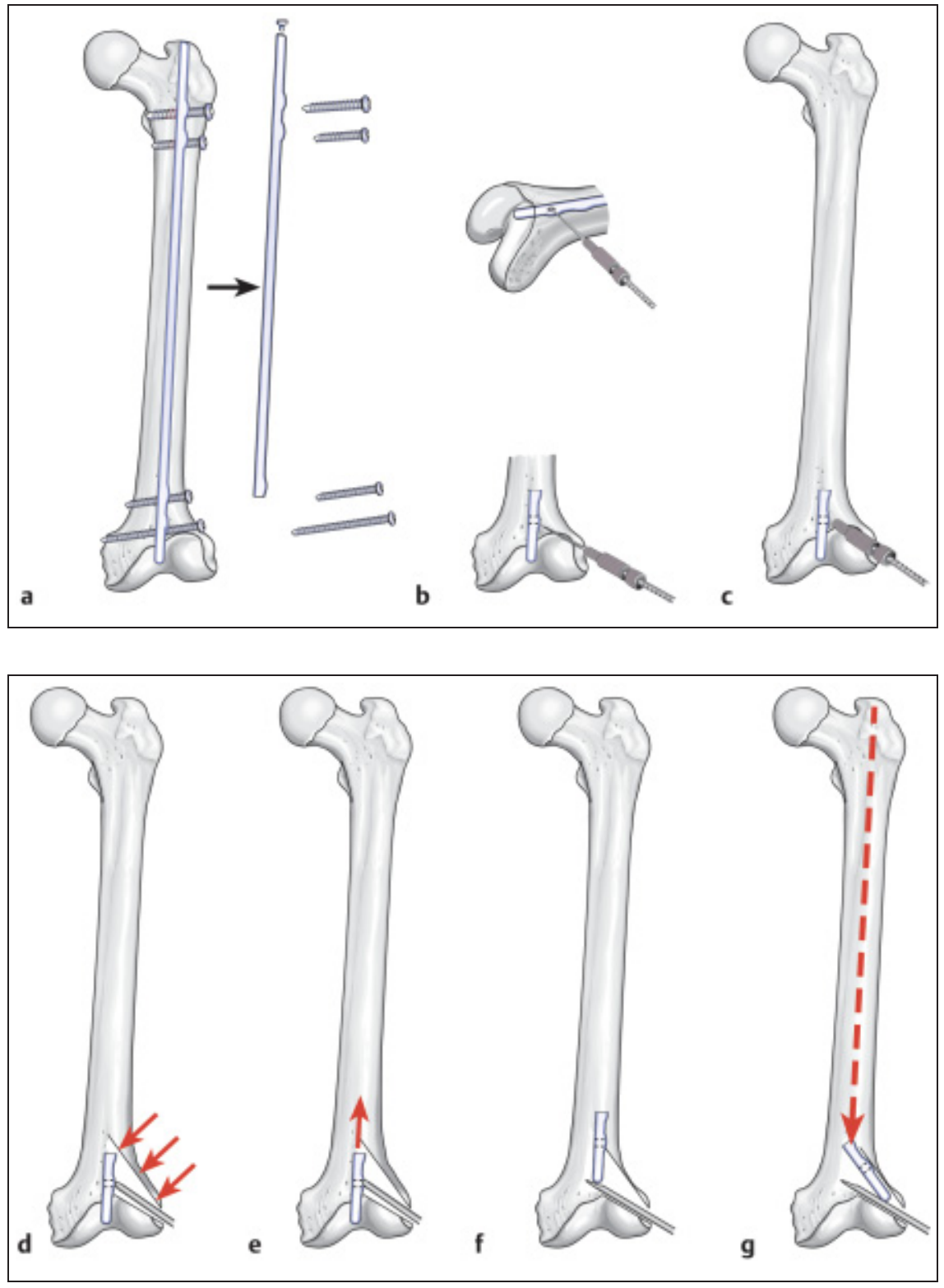

Abb. 7a-g Schematische Zeichnungen zur Erläuterung der Methode zum Entfernen des distalen Fragments eines soliden, femoralen Nagels, der auf Höhe der Proximaleren von den zwei distalen Schraubenlöchern gebrochen war. Weiteres siehe Text.

des linken Beines an Unterarmgehstützen für 6 Wochen, und anschließend mit der Hälfte des Köpergewichts für 3 Wochen mobilisiert. Die Fraktur war nach 4 Monaten radiologisch durchbaut. 16 Monate nach der Operation bestand Schmerzfreiheit und ein normaler Bewegungsumfang des Kniegelenks.

Ein Nachteil dieser Technik ist, dass sie nicht verwendet werden kann, um lange Fragmente eines zerbrochenen Nagels zu entfernen. Bei Bruch eines Nagels auf Höhe der proximalen Schraubenlöcher oder der Fraktur kann aber in umgekehrter Richtung (von distal nach pro- ximal) das Fragment herausgeschoben werden. Denkbar ist auch die anterograde, transartikulär-interkondyläre Tech-

Lösungsmöglichkeiten bei Repositionsverlust

Die zunehmende Verschiebung der Altersstruktur unserer Bevölkerung bringt es mit sich, dass Osteoporose bedingte Verankerungsprobleme zunehmend auch bei Verriegelungsnagelosteosynthesen beachtet werden müssen. Die Verriegelungsbolzen stützen sich bei hochgradiger Osteoporose in den Metaphysen von Femur und Tibia gelegentlich nur unzureichend ab, was erhebliche Verankerungsprobleme mit sich bringt. Für dieses Problem bei ante- und retrograder Nagelungen haben wir spezielle Modularschrauben ( $\mathrm{Fa}$. ARGE, Medizintechnik, Hannover) entwickelt, die über ein Führungsdrahtsystem auf konventionelle Verriegelungsbolzen aufgeschraubt werden (Abb. 8a-c). Damit wird eine größere Auflagefläche und verbesserte Abstützung im distalen Femur erzielt und das Risiko einer Bolzenwanderung in kraniokaudaler Richtung reduziert. Zusätzlich wird die Kippstabilität des Nagels erhöht. Das Innengewinde in der Modularschraube vermindert das Risiko einer Bolzenmigration in mediolateraler Richtung. Die Platzierung erfolgt in folgenden Schritten: Nach Nagelimplantation 1. Bohren der Verriegelungslöcher und 2. Einführen des Führungsdrahts für die Modularschraube, 3. Bohren und Gewindeschneiden für das Modularschraubengewinde $(9,5 \mathrm{~mm}), 4$. Einschrauben der Modularschrauben von medial und lateral und 5. als letzter Schritt Einschrauben des Verriegelungsbolzens. Die Modularschrauben können auf Kortikalisniveau eingedreht werden und führen deshalb zu keiner Weichteilirritation.

Im metaphysennahen Bereich besteht eine zunehmende Diskrepanz zwischen Implantat- und Markraumdurchmesser. Diese fehlende Abstützung wird durch die Verriegelungsbolzen oft nicht ausreichend aufgefangen (mechanisches Spiel zwischen Bolzen und Nagel, Bolzenverformung und Translokation des Nagels auf den Bolzen). Die Folgen sind Achsenfehler und/oder knöcherne Konsolidierungsstörungen [9]. Ein internes Zusatzstabilisierungskonzept wurde entwickelt [7], mit dem metaphysennahe Frakturen mit zusätzlichen abstützenden Implantaten „nagelfähig“ gemacht werden. Dabei werden nagel- und frakturnah platzierte Verriegelungsbolzen (Pollerschrauben) mit dem Ziel der Implantatabstützung eingebracht. Diese Pollerschrauben haben eine sperrende Wirkung für Translationen senkrecht zur Nagellängsachse, verhindern so sekundäre Dislokationen und erhöhen die Primärstabilität des Knochenimplantatverbunds, ohne dass eine axial sperrende Wirkung besteht. Sie werden in der Regel vor der Nagelimplantation entsprechend einer präoperativen Planung möglichst frakturnah eingebracht, der anschließend eingebrachte Nagel gleitet daran vorbei und stützt sich an der Pollerschraube ab (Abb.9au.b). 
a

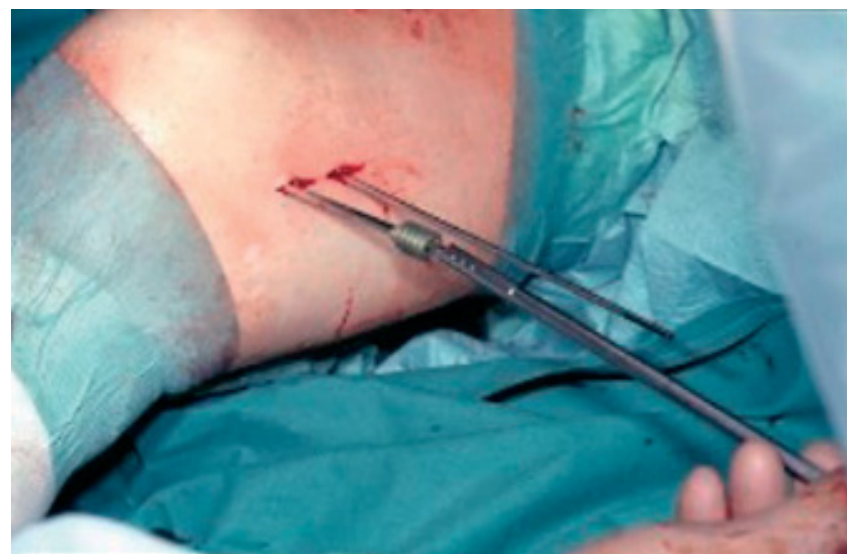

b, c
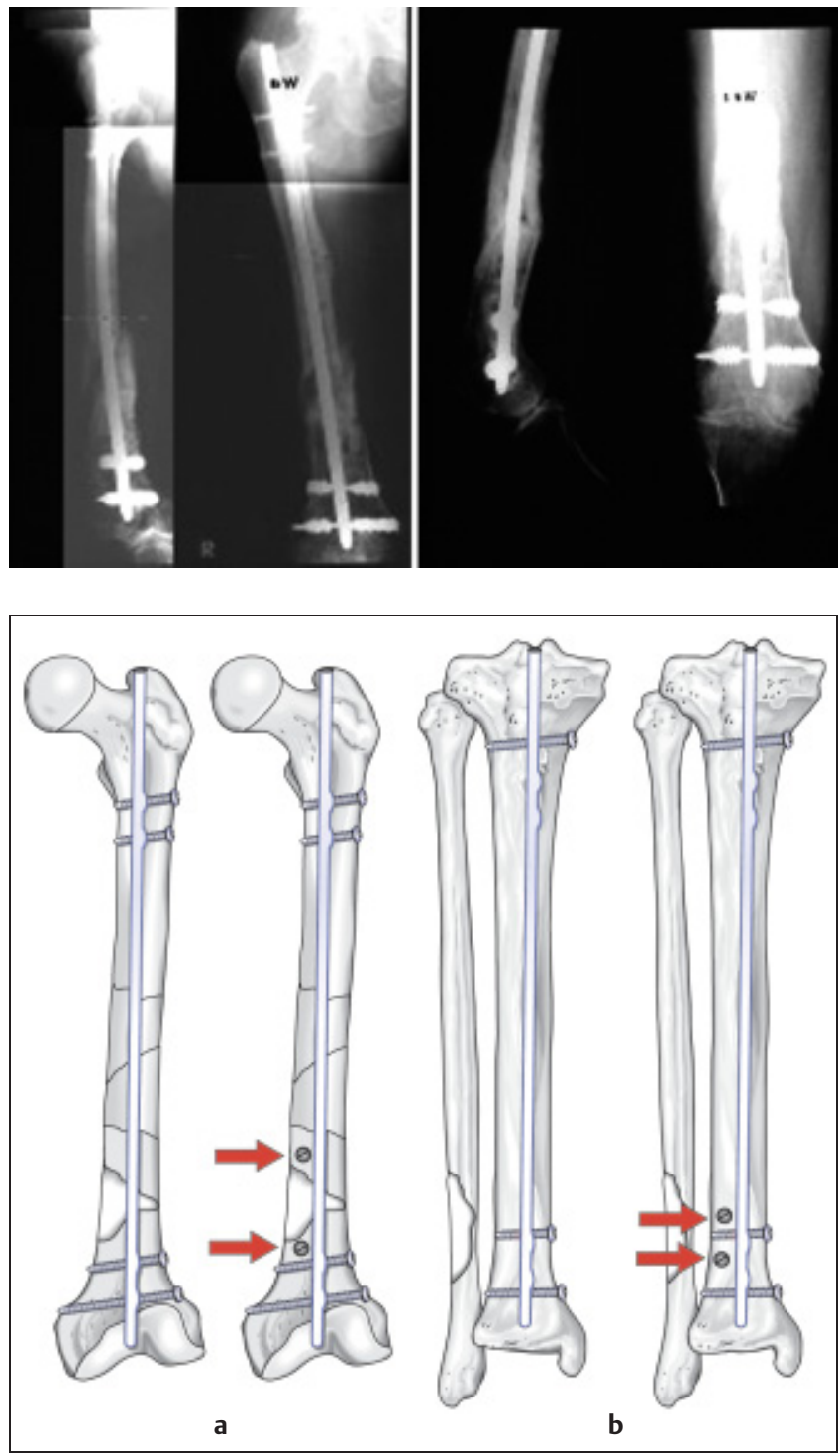

Abb.9a u. b Pollerschrauben bei der intramedullären Osteosynthese von distalen Femur- und Tibiaschaftfrakturen. (a) Schematische Darstellung der räumlichen Verhältnisse am distalen Femur und (b) distaler Tibia. Missverhältnis zwischen Implantataußen- und Kortikalisinnendurchmesser. Dadurch kann die Implantabstützung ungenügend sein mit der Folge von Instabilität und/oder Fehlstellung. Durch die Verwendung von implantatnah eingebrachten Pollerschrauben erfolgt eine wirksame Abstützung. Damit können Instabilität und/oder Fehlstellung vermieden werden.
Abb. 8a-c Neue Verankerungstechnik von Verriegelungsbolzen bei schwerer Osteoporose (Modularschrauben). (a) Insertion der kanülierten Modularschrauben über Führungsdraht von lateral und medial. (b, u.c) Klinischer Fall mit schwerer Osteoporose und multiplen vorausgegangenen Eingriffen bei erneuter Fraktur nach Sturz in der AHB. Verlaufskontrolle nach 6 Wochen, Fraktur nach 18 Wochen verheilt.
Durch die Anwendung von Pollerschrauben können vorbestehende, sklerosierte Kanäle nach vorangegangener Nagelung in Fehlstellung ebenso kompensiert werden, wie die häufig zu Fehlstellungen neigenden, schwierig zu versorgenden proximalen Tibiafrakturen.

Die Abb.10a -e stellen eine Kombination aus den zuvor beschriebenen Problembereichen dar. Nach initialer Versorgung durch einen unverriegelten Universalnagel auswärtig erfolgte die Vorstellung des Patienten bei uns. Der Nagel war inzwischen gebrochen und die Fraktur war mit einer Verkürzung von $6 \mathrm{~cm}$ rechts ausgeheilt. Zudem bestand ein Innenrotationsfehler am Oberschenkel. Nach der Implantatenfernung und Korrekturosteotomie erfolgte die Stabilisierung durch einen unaufgebohrten Verriegelungsmarknagel in Kombination mit einem externen unilateralen Fixateur. Über diesen erfolgte über 4 Monate die Distraktion des Oberschenkels. Periphere Nervenschäden erlitt der Patient dabei nicht. Nach Abnahme des Fixateurs erfolgte zur Korrektur der Varusfehlstellung das Setzen von Pollerschrauben im Bereich der medialen Kortex im jeweils oberen und unteren Ende der Hauptfragmente, um eine erneute Varusfehlstellung durch Wandern des Nagels nach medial zu verhindern.

\section{Schlussfolgerung}

Im Rahmen der Versorgung von Schaftfrakturen am Oberschenkel durch intramedulläre Stabilisierungsverfahren sind die korrekte Einstellung der Achsen in der Frontal- und Sagittalebene und die richtige Zugangswahl für den Erfolg der Osteosynthese grundlegend. Nachträgliche Eingriffe zur Korrektur von Repositionsverlusten und damit verbunden häufig auch die Entfernung vorhandener Implantate sind wie wir gezeigt haben durchführbar, erfordern aber meist größere physische und psychische Ressourcen als beim Primäreingriff. Durch innovative Zusatzimplantate wie die Modular- und Pollerschrauben ist eine Lösung von Fehlschlägen mit relativ geringem Aufwand durchführbar. 

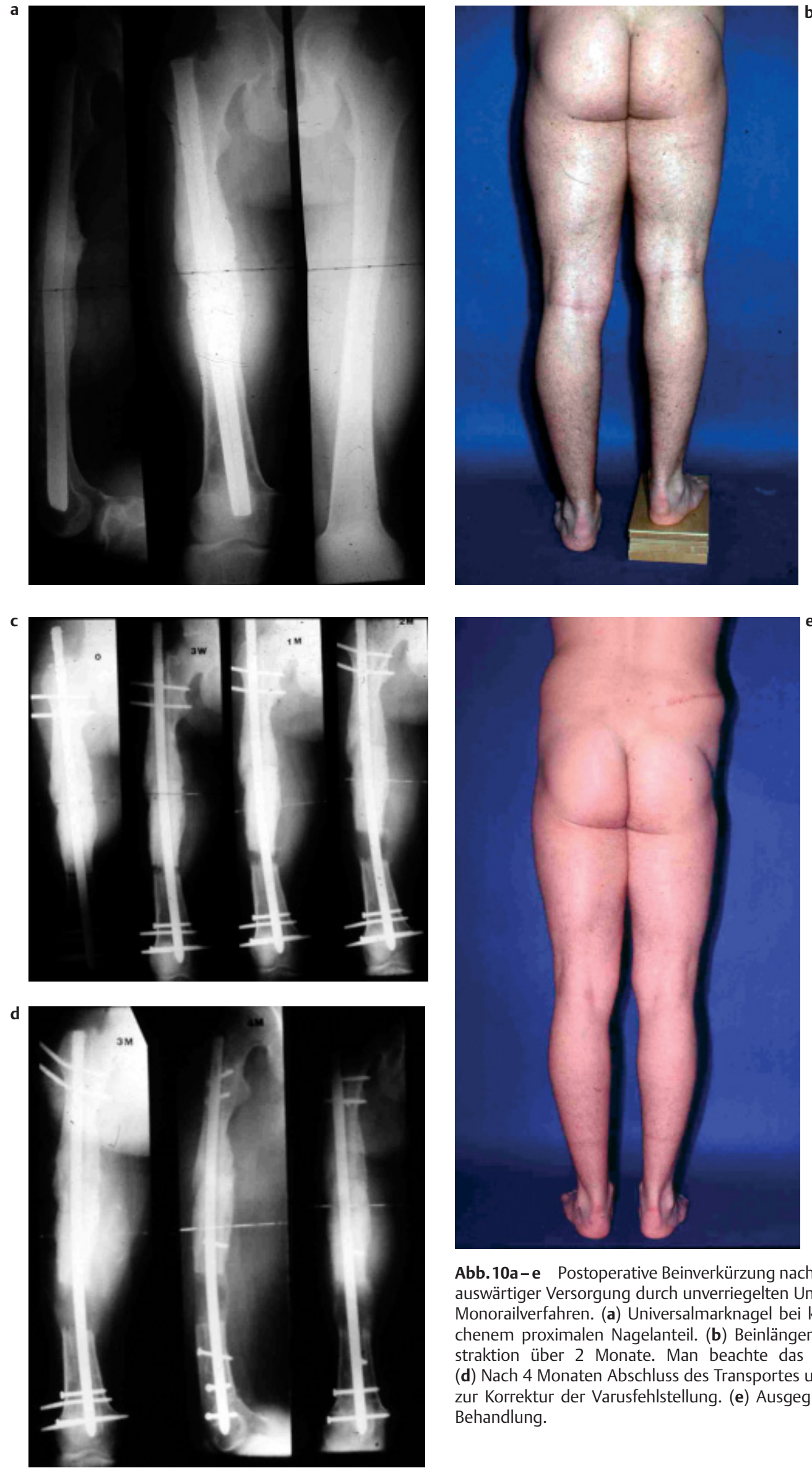

Abb.10a-e Postoperative Beinverkürzung nach Oberschenkelschaftfraktur rechts und auswärtiger Versorgung durch unverriegelten Universalmarknagel. Verlängerung durch Monorailverfahren. (a) Universalmarknagel bei konsolidierter Fehlstellung und gebrochenem proximalen Nagelanteil. (b) Beinlängenverkürzung $6 \mathrm{~cm}$ rechts. (c) Kallusdistraktion über 2 Monate. Man beachte das Durchbiegen der Schanz-Schrauben. (d) Nach 4 Monaten Abschluss des Transportes und Setzen von Pollerschrauben medial zur Korrektur der Varusfehlstellung. (e) Ausgeglichene Beinlänge nach Abschluss der Behandlung. 


\section{Literatur}

${ }^{1}$ Browner BD. Pitfalls, errors and complications in the use of locking Küntscher nails. Clin Orthop 1986; 212: 192-208

${ }^{2}$ Browner BD. The science and practice of intramedullary nailing. 2. Auflage. Williams and Wilkins, Balitmore 1996

${ }^{3}$ Buchholz RW, Ross SE, Lawrence KL. Fatigue fracture of the interlocking nail in the treatment of fractures of the distal part of the femoral shaft. J Bone J Surg 1987; 69-A: 1391 1399

${ }^{4}$ Kempf I, Grosse A, Abalo C. Locked intramedullary nailing: its application to femora and tibial axial rotational, lengthening, and shortening osteotomies. Clin Orthop 1986; 212: $165-73$
${ }^{5}$ Krettek C, Miclau T, Grun O, Schandelmaier $\mathrm{P}$, Tscherne H. Intraoperative control of axes, rotation and length in femoral and tibial fractures. Technical note. Injury 1998; 29 Suppl 3: C29-39

${ }^{6}$ Krettek C. Prinzipien der intramedullären Knochenbruchstabilisierung. Unfallchirurg 2001; 104: 639-653

${ }^{7}$ Krettek C, Schulte-Eistrup S, Schandelmaier $\mathrm{P}$, Rudolf J, Tscherne H. Osteosynthese von Femurschaftfrakturen mit dem unaufgebohrten AO-Femurnagel (UFN): Operationstechnik und erste klinische Ergebnisse mit Standardverriegelung. Unfallchirurg 1994; 97: $549-567$

${ }^{8}$ Levy O, Amit Y, Velkes S, Horoszowski H. A simple method for removal of a fractured intramedullary nail. J Bone J Surg 1994; 76B(3): 502
${ }^{9}$ Tornetta P Tiburzi D Reamed vs, nonreamed femoral nailing: a preliminary report. Orthopaedic Trauma Association (OTA), Annual Meeting Boston 27. - 29. 9. 1996, Abstractbook, 1996: pp 28-29

${ }^{10}$ Winquist RA, Hansen ST Jr, Clawson DK Closed intramedullary nailing of femora fractures. A report of five hundred and twenty cases. J Bone J Surg 1984; 66-A 529-539

\section{Dr. med. Ulrich Wiebking}

Assistenzarzt

Prof. Dr. med. Christian Krettek FRACS

Direktor der Klinik f. Unfallchirurgie

Medizinische Hochschule Hannover Carl-Neuberg-Str. 1

D-30625 Hannover 